\title{
Synapse Distribution on VCH, an Inhibitory, Motion-Sensitive Interneuron in the Fly Visual System
}

\author{
VOLKER GAUCK, 1,* MARTIN EGELHAAF, ${ }^{2}$ AND ALEXANDER BORST ${ }^{1}$ \\ ${ }^{1}$ Friedrich-M iescher-Laboratorium der Max-Planck-Gesellschaft, \\ D-72076 Tübingen, Germany \\ 2Lehrstuhl für Neurobiologie, F akultät für Biologie, Universität Bielefeld, \\ D-33501 Bielefeld, Germany
}

\begin{abstract}
In this study, the distribution of synapses in the ventral centrifugal horizontal (VCH)-a nonspiking, inhibitory, motion-sensitive interneuron in the third visual ganglion (lobula plate) of the blowfly Calliphora erythrocephala-was examined by electron microscopy and electrophysiology. The frequency histograms of excitatory and inhibitory postsynaptic potential amplitudes recorded from the $\mathrm{VCH}$ during contralateral stimulus presentation suggest the existence of three neurons, two excitatory and one inhibitory, mediating contralateral input. To localize input and output regions of the $\mathrm{VCH}$, we investigated the ultrastructure of its two arborisation areas after intracellular iontophoretic injection of horseradish peroxidase. The VCH has input synapses in its arborisation in the protocerebrum and in its arborisation in the Iobula plate. Output synapses were found exclusively in the lobula plate. Thus, the large dendritic arbor of the VCH in the lobula plate serves simultaneously as an input and an output region. There, we found input and output synapses in close vicinity $(0.5-1.5 \mu \mathrm{m})$ to each other. Taking into account that the $\mathrm{VCH}$ receives retinotopicly arranged input from the ipsilateral eye in the lobula plate, the close location of input and output synapses in the VCH suggests that the spatial organization of its retinotopic synaptic input is more or less conserved in its inhibitory output pattern. The VCH has been proposed to inhibit the figure detection 1 (FD1), another neuron of the lobula plate, that responds preferentially to small moving objects. These results suggest that the FD1 may receive inhibitory inputs from the $\mathrm{VCH}$ in the lobula plate, where the dendritic arbors of both neurons overlap.J . Comp. Neurol. 381:489-499, 1997.
\end{abstract}

Indexing terms: Calliphora erythrocephala; lobula plate; figure-ground discrimination; electron microscopy; electrophysiology

Objects in a visual scene can be discerned from the background by many different cues. They might have a different brightness, color, texture, or depth than the background. When the observer is freeto move in the environment, motion information also can be used to segregate the environment into objects and their background. This cue can be used for figure-ground discrimination, because the retinal images of objects and background usually move at different vel ocities. By comparing behavioral and electrophysiologic data obtained under the same stimulus conditions, some neurons of the fly visual system - the so-called figure detection (FD) cells-have been shown to play a dedsive role in mediating figure-ground discrimination behavior (Egelhaaf, 1985a,b,c, 1987; Egelhaaf et al., 1988; Reichardt et al., 1989).

So far, four FDs have been characterized. They bel ong to a set of approximately 50-60 tangential neurons located in the posterior part of the third visual neuropil, the lobula plate. All tangential neurons studied so far respond to motion stimuli in a directionally selective way. Most tangential neurons, presumably induding the FDs, with their large almost planar dendrites, spatially integrate synaptic input from retinotopically arranged neurons originating in the medulla and lobula (Strausfeld, 1984; Borst and Egel haaf, 1992; Egelhaaf and Borst, 1995). Thetangential neurons differ with respect to their preferred direction (Hausen, 1984) and their mode of action (Haag and Borst,

*Correspondence to: Dr. Gauck, Friedrich-Miescher-Laboratorium der Max-Planck-Gesellschaft, Spemannstrasse 37-39, D-72076 Tübingen, Germany. E-mail: gauck@sunwan.mpik-tueb.mpg.de

Received 9 May 1996; Revised 23 December 1997; Accepted 8 J anuary 1997 
1996). Some tangential neurons respond to motion stimulation exclusively with graded potential shifts. Others (like the FDs) are spiking neurons, whereas a third class of neurons responds in an intermediate mode consisting of graded potential shifts with superimposed spike-like events. The FDs differ from all other tangential neurons studied so far in that they respond better to small than to large stimuli. When the stimulus pattern exceeds a certain size, the response of the FDs decreases (E gel haaf, 1985c). This observation suggests that inhibitory elements exist that mediate the tuning of the FDs to small objects (Egelhaaf, 1985a,c). In the case of the figure detection 1 (FD1), the ventral centrifugal horizontal (VCH)-a neuron responding exclusively with graded membrane potential changes (Hausen, 1977)- has been suggested as the relevant inhibitory neuron (Warzecha et al., 1993) by using a photoinactivation technique developed by Miller and Selverston (1979). This is in accordance with pharmacologic evidence. Application of picrotoxinin, an antagonist of the inhibitory transmitter $\gamma$-aminobutyric acid (GABA), also el iminates the characteristic small-field tuning of the FD1 (Egelhaaf, 1990; Warzecha et al., 1993). Indeed, the VCH and its companion neuron the dorsal centrifugal horizontal $(\mathrm{DCH})$ are thought to be GABA-ergic on the basis of histochemical investigations (Meyer et al., 1986).

The $\mathrm{VCH}$ and $\mathrm{DCH}$ are very similar in their anatomical and el ectrophysiologic properties. They are depolarized by front-to-back motion in front of the ipsilateral eye and hyperpolarized by motion in the opposite direction (Hausen, 1977). Back-to-front motion in front of the contralateral eye elicits excitatory postsynaptic potentials (EPSPs), and motion in the opposite direction elicits inhibitory postsynaptic potentials (IPSPs; Hausen, 1977). The VCH and $\mathrm{DCH}$ differ with respect to their ipsilateral receptive fields. The VCH responds to motion in the lower part of the ipsilateral visual field, and the DCH responds to motion in the upper part. The VCH covers the ventral part of the lobula plate and the DCH the dorsal part (Hausen, 1977). Both neurons possess very fine processes that cover the lobula plate, almost homogeneously, from the proximal to distal ends (Hausen, 1977). In addition, these cells have a second area of arborisation in the ipsilateral dorsal protocerebrum with short, dense processes. Both fields of arborisation are connected by a presumed "axon." Their somata are on the contralateral side, dorsal to the oesophagus foramen. They are connected to the protocerebral arborisation by a thin neurite. Calcium imaging experiments (Egelhaaf et al., 1993) revealed that the $\mathrm{VCH}$ receives excitatory input from contralateral neurons in the lobula plate and in the protocerebrum. These recordings also revealed that, in the lobula plate, the $\mathrm{VCH}$ receives retinotopically arranged input from the ipsilateral eye. In addition the $\mathrm{VCH}$ receives inhibitory input from contralateral neurons. These findings indicate that the $\mathrm{VCH}$ receives synaptic input in the lobula plate and in the protocerebrum. Hausen $(1976,1977)$ mentioned varicosities ("blebs") in the lobula plate arborisation of the VCH possibly indicative of output synapses. There is also a note in Eckert and Meller (1981) that the VCH is presynaptic and postsynaptic in the lobula plate, but ultrastructural data concerning the synaptic organization of the $\mathrm{VCH}$ have not been presented. The arborisations of the VCH and FDI overlap in the ventral and distal fourth of the Iobula plate, raising the possibility that the FD1 is inhibited by the $\mathrm{VCH}$ in the lobula plate.
It is supposed that the location of the inhibitory synapses that the FD1 receives from the $\mathrm{VCH}$ may be crucial for the physiologic implementation of small-field tuning (Borst and Egel haaf, 1993). Theaim of the present study is to elucidate this point further. Therefore, we investigated the synaptic input and output organization of the VCH by electron microscopy.

\section{MATERIALS AND METHODS Preparation}

Experiments were performed with 1- to 3-day-old female blowflies, Calliphora erythrocephal a, obtained from laboratory stocks of the Max-Planck-I nstitute of Biol ogical Cybernetics. Flies were mounted with the dorsal side of the thorax glued to a small piece of glass. The head capsule was opened from behind. To allow access to the lobula plate, airsacs and tracheas were removed. The symmetry of the deep pseudopupil (Franceschini and Kirschfeld, 1971) of both eyes was used to adjust the animals to the center of the stimulation device.

\section{E lectrophysiologic recording}

For intracellular recording, glass mi cropipettes (GC100F10; Clark, Electromedical, Reading, UK) were pulled on a Brown-Flaming micropipette puller (P-87; Sutter Instruments, Norato, CA). Electrode tips were backfilled with $4 \%$ Lucifer yellow (LY) in $1 \mathrm{M} \mathrm{LiCl}$, and el ectrode shafts were filled with $1 \mathrm{M} \mathrm{LiCl}$. The electrodes had resistances of 50-70 $\mathrm{M} \Omega$. At the end of the recording, cells were labelled by injection of LY. To label cells for electron microscopy, electrode tips were backfilled with a solution of $4 \%$ horseradish peroxidase (Boehringer, Mannheim, Germany) in $0.1 \mathrm{M} \mathrm{Tris} / 0.5 \mathrm{M} \mathrm{KCl}$. Electrode shafts were filled with $0.5 \mathrm{M} \mathrm{KCl}$, resulting in resistances of $150-230 \mathrm{M} \Omega$. Recorded signals were amplified by the use of standard electrophysiologic equipment and fed through an $A / D$ converter (DT 2801-A; Data Translation, Marlboro, MA) into an IBM (Mainz, Germany) PS2 computer at a sampling rate of $3.3 \mathrm{kHz}$.

\section{Visual stimulation}

While recording from the $\mathrm{VCH}$, the flies were visually stimulated. The animals were positioned in the center of a cylindrical stimulus device of $240^{\circ}$ horizontal angular extent. Seen from the animal's perspective, the stimulus pattern moved horizontally in front of a cylindrical frosted glass, behind which the background was illuminated by eight neon lights. Seven opaque, equally spaced, vertically oriented stripes formed a pattern of $50^{\circ}$ horizontal extent. All cells were recorded in the right half of the brain so that clockwise motion (looking onto the preparation) corresponded to motion in the preferred direction of $\mathrm{VCH}$. Azimuthal stimulus positions to the right or the left of the midsagittal plane of the fly are given as positive or negative values. Accordingly, stimulus positions above or below the equatorial plane are given as positive or negative values. The stimulus pattern extended from $-60^{\circ}$ to $+60^{\circ}$ vertically. The center of the pattern moved from $-100^{\circ}$ to $-60^{\circ}$ and back to $-100^{\circ}$ (in front of the contralateral eye) and from $60^{\circ}$ to $100^{\circ}$ and back to $60^{\circ}$ (in front of the ipsilateral eye) in a sinusoidal way. The pattern contrast was $96 \%$. The mean luminance of the pattern was approximately $90 \mathrm{~cd} / \mathrm{m}^{2}$. 


\section{contralateral}
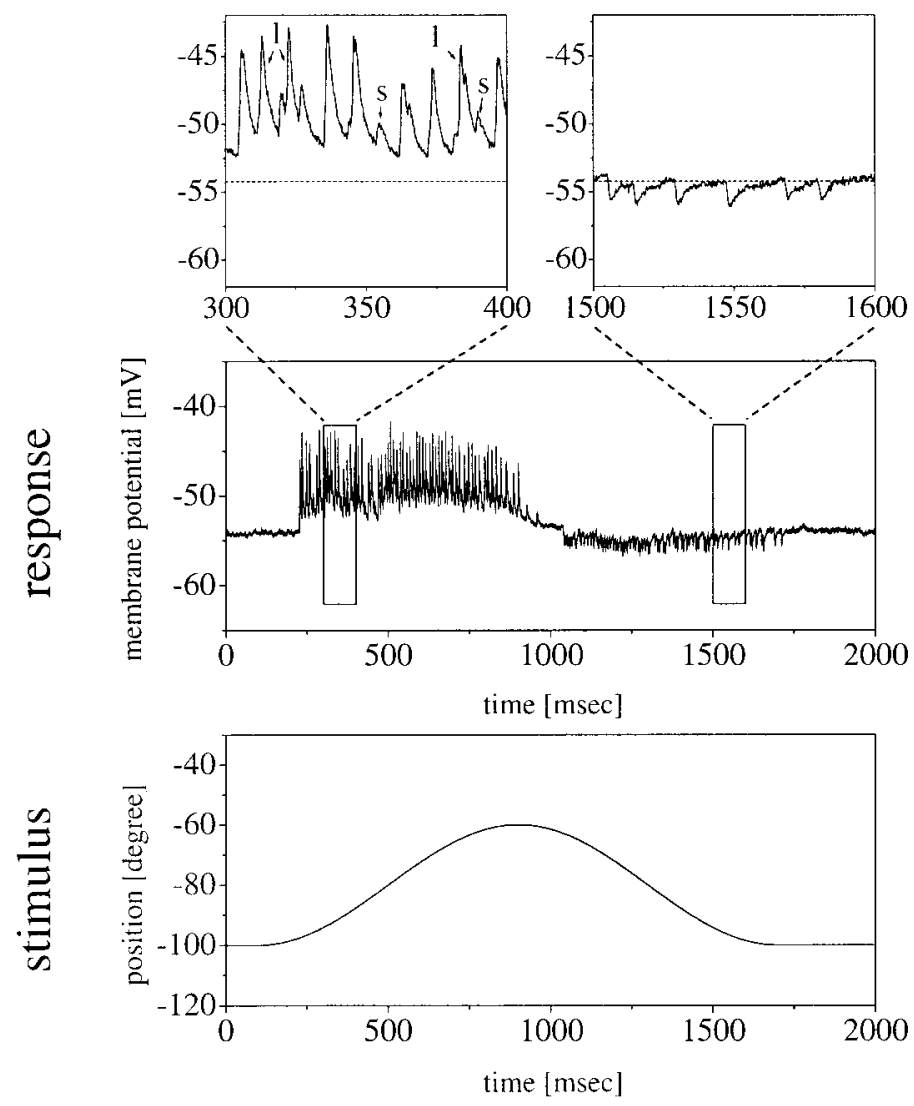

\section{ipsilateral}
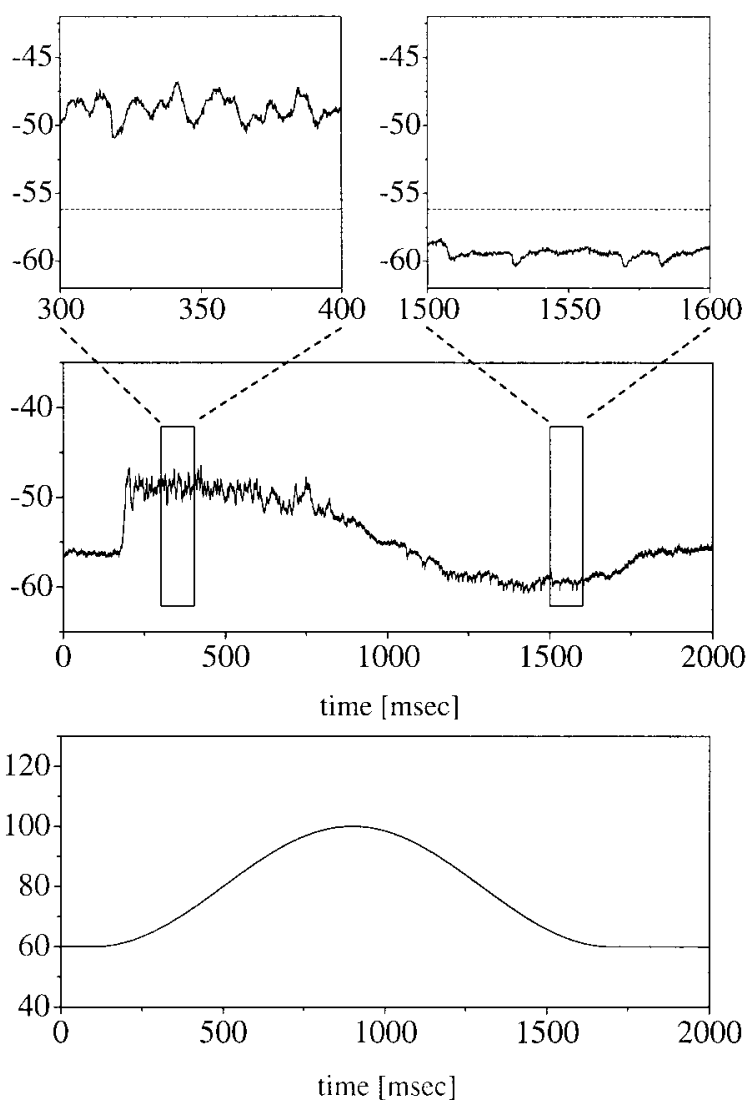

Fig. 1. Responsetraces of an identified ventral centrifugal horizontal (VCH, Lucifer yellow injection). The bottom row shows the stimulus position as a function of time. The stimulus pattern $\left(50^{\circ}\right.$ total angular extent) moved clockwise (toward higher values) and counterclockwise (toward lower values) in front of the contralateral eye (left column) and in front of the ipsilateral eye (right column). The middle row shows the membrane potential of the VCH due to motion stimulation as recorded in the axon of the cell. In the upper row the membrane potential of the $\mathrm{VCH}$ during visual stimulation is shown at

\section{Cell identification}

The identification of $\mathrm{VCH}$ was based on the following characteristic physiol ogic responses to motion stimuli (Hausen, 1977; E ckert and Dvorak, 1983): 1) graded membrane potential changes without spikes, 2) maximum sensitivity in the ventral part of the ipsilateral visual field, 3) depolarization in response to motion from the front to the back in the ipsilateral visual field, and hyperpolarization in response to motion in the opposite direction (Fig. 1, right), 4) EPSPs in response to motion from the back to the front in the contralateral visual field, and IPSPs in response to motion in the opposite direction (Fig. 1, left). Typical response traces showing the characteristics of VCH membrane potential under the visual stimulus arrangements described above are shown in Figure 1.

\section{E lectronmicroscopy}

$\mathrm{VCH}$ was recorded as described above. After identification of a VCH based on its response characteristics, horseradish peroxidase (HRP) was injected for 2-20 min- higher time resolution. The resting membrane potential averaged during the first $100 \mathrm{msec}$ is plotted as a dotted line. Clockwise motion in front of the contralateral eye elicits excitatory postsynaptic potentials (EPSPS) but does not in the case of motion in front of the ipsilateral eye. Small (s) and large (I) EPSPs can be discerned qualitatively. Counterclockwise motion in front of the contralateral eye elicits inhibitory postsynaptic potentials (IPSPs), without shifting the membrane potential to more negative values, as counterclockwise motion in front of the ipsilateral eye does.

utes by a constant depolarizing current of 1-2 nA. After diffusion of HRP for 1 hour at $4^{\circ} \mathrm{C}$, the tracheae and lipid bodies were removed from the head capsule. The head of the fly was cut off and fixed for 3 hours at $4^{\circ} \mathrm{C}$ in $4 \%$ paraformal dehyde and $0.3 \%$ glutaral dehyde mixture in 0.2 $\mathrm{M}$ phosphate buffer with $1 \%$ sucrose, $\mathrm{pH} 7.4$. The brain was rinsed for 3 hours at $4^{\circ} \mathrm{C}$ in $0.05 \%$ diaminobenzidine (DAB) and $0.002 \% \mathrm{NiNH}_{4} \mathrm{SO}_{4}$ in $0.2 \mathrm{M}$ phosphate buffer with $1 \%$ saccharose, $\mathrm{pH}$ 7.4. Then, the $\mathrm{DAB}$ reaction was started by transferring the brain in $0.01 \% \mathrm{H}_{2} \mathrm{O}_{2}$ and $0.05 \%$ DAB and $0.002 \% \mathrm{NiNH}_{4} \mathrm{SO}_{4}$ in $0.2 \mathrm{M}$ phosphate buffer with $1 \%$ saccharose, $\mathrm{pH} 7.4$. The stained cells were then inspected with the light microscope and identified based on their characteristic dendritic arborisation pattern (see Fig. 3). For electron microscopic investigation, we exclusively used brains in which only $\mathrm{VCH}$ was stained. The preparations were placed in 1\% osmium tetroxide for 1 hour at room temperature. Afterward, the brain was taken out of the head capsule, dehydrated, and embedded in Epon (Serva, Heidelberg, Germany). Ribbons of serial 


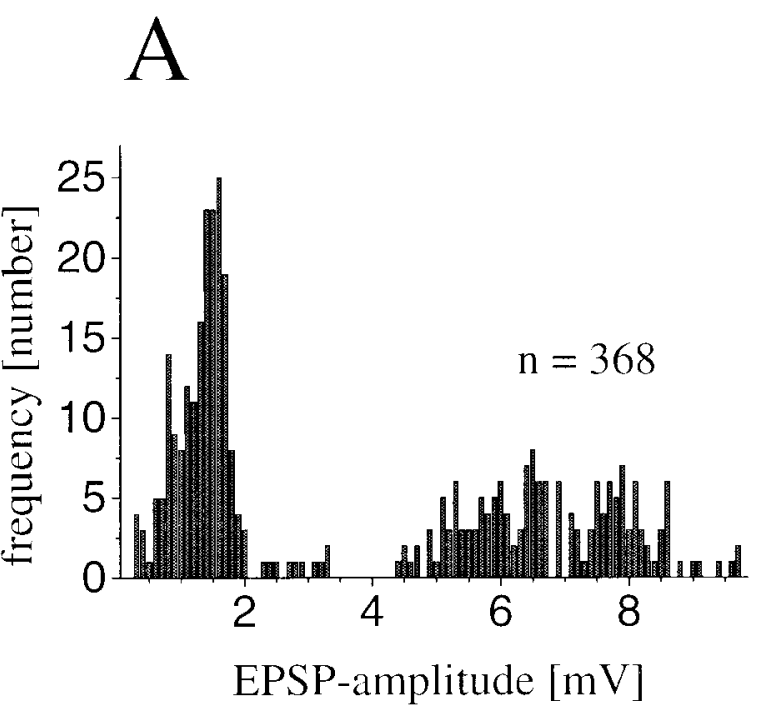

Fig. 2. (A) EPSP and (B) IPSP amplitudes recorded from an identified VCH (Lucifer yellow injection) during motion stimulation in front of the contralateral eye. $\mathrm{N}$ gives the total number of counted postsynaptic potentials (PSPs). The absolute number of PSPS is plotted against their amplitude (bin-width $=0.1 \mathrm{mV}$ ). The PSP ampli-

sections were cut with a diamond knife on an ultramicrotome (UItracut, Leica, Bensheim, Germany) and collected on Formvar (Serva, Heidelberg, Germany) coated slot grids (Plano, Wetzlar, Germany). Sections were contrasted with uranyl acetate and lead citrate before examination on a Zeiss (Oberkochen, Germany) 9 S-2 el ectron microscope.

\section{RE SULTS E lectrophysiology}

Depending on the direction of motion, $\mathrm{VCH}$ receives excitatory and inhibitory input from both eyes (Fig. 1). $\mathrm{VCH}$ is excited by back-to-front motion and front-to-back motion in the contralateral and ipsilateral visual fields, respectively. It is inhibited by motion in therespectiveopposite directions. To analyze the number of excitatory and inhibitory contralateral input elements of $\mathrm{VCH}$, we determined the amplitude spectra of EPSPs and IPSPs of one identified VCH during visual motion in front of the contralateral eye, an experiment previously performed by Hausen (1977) for unidentified centrifugal horizontal $(\mathrm{CH})$ neurons $(\mathrm{VCH}$ or $\mathrm{DCH}$ ). Back-to-front motion in front of the contralateral eye elicits EPSPs of different amplitude. Small and large EPSPs can be discerned qualitatively (Fig. 1 upper left). The frequency histogram in Figure 2A shows two groups of EPSP amplitudes. The first group shows a clear peak at $1.6 \mathrm{mV}$. The second group is broadly tuned with a peak about $6.5 \mathrm{mV}$. This result suggests that $\mathrm{VCH}$ receives synaptic input from two contralateral neurons. The broad tuning of the second group is probably due to temporal summation of large and small EPSPs. The amplitude spectrum of IPSPs in Figure 2B shows one single peak at $1.7 \mathrm{mV}$, suggesting that contralateral inhibitory input is mediated by one neuron.

\section{Electron microscopy}

To characterize the connectivity between VCH and FD1, we investigated the ramifications of $\mathrm{VCH}$ in the lobula

\section{B}

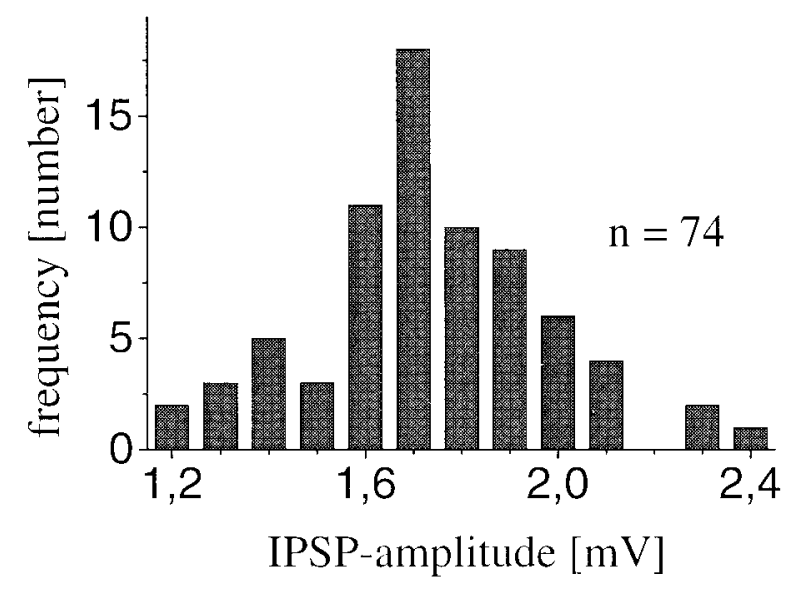

tude was measured between the basis of the steep upstroke or downstroke, respectively, and their peaks. Two amplitude groups of EPSPs can be discerned (A), suggesting that contralateral excitatory input is mediated by two neurons. IPSPs show only one peak (B), indicating that one neuron mediates contralateral inhibition.

plate and in the protocerebrum with an electron microscope. VCH was labelled by intracellular iontophoretic injection of HRP followed by a DAB reaction. Synapses of $\mathrm{VCH}$ were identified by the presence of the typical Tshaped presynaptic ribbons, which have been found in the opticlobes of thefly species Calli phora and Musca (CamposOrtega and Strausfeld, 1973; Burkhardt and Braitenberg, 1976; Hausen et al., 1980; Shaw and Meinertzhagen, 1986).

Figure 3 illustrates the appearance of an HRP-injected $\mathrm{VCH}$ in the light microscope, showing its dense lobula plate arborisation pattern, the axon, the protocerebral arborisation, the cell body fibre, and the soma. Several electron micrographs of the lobula plate are shown in Figure 4. Cross-sections of $\mathrm{VCH}$ arbors are dark-labeled by the DAB polymerization product. In Figure $4 \mathrm{~A}$, the characteristic presynaptic ribbon (arrowhead) in close contact to $\mathrm{VCH}$ arbor indicates an input synapse (I) of $\mathrm{VCH}$. At this synapse, a second unknown element at the left to $\mathrm{VCH}$ receives identical input.

Figure 4B shows another example of an input synapse onto the lobula plate arborisation of $\mathrm{VCH}$. Here, two other cells share this presynaptic site with $\mathrm{VCH}$. In general, we found up to four other unstained postsynaptic elements at input synapses of $\mathrm{VCH}$ in the lobula plate. Figures $4 \mathrm{C}, \mathrm{D}$, and $E$ represent three successive sections of an input synapse of VCH taken at 60- to 80-nm distance. At one end of the presynapticribbon where only its plateis visible (arrowhead I, Fig. 4C), two unstained elements are in contact with the presynapse (arrowheads 1 and 2), but VCH has no contact. In Figure 4D, where the whole presynaptic ribbon can be seen (arrowhead I), VCH is in contact with the presynaptic site. The unstained postsynaptic element 1 remains in the same position as in Figure 4C, but on the arbor of $\mathrm{VCH}$ the picture has changed. Two smaller elements are visible, from which presumably the smaller one (arrowhead 3 ) is not yet present in Figure $4 \mathrm{C}$, and the bigger one (arrowhead 2 ) is 


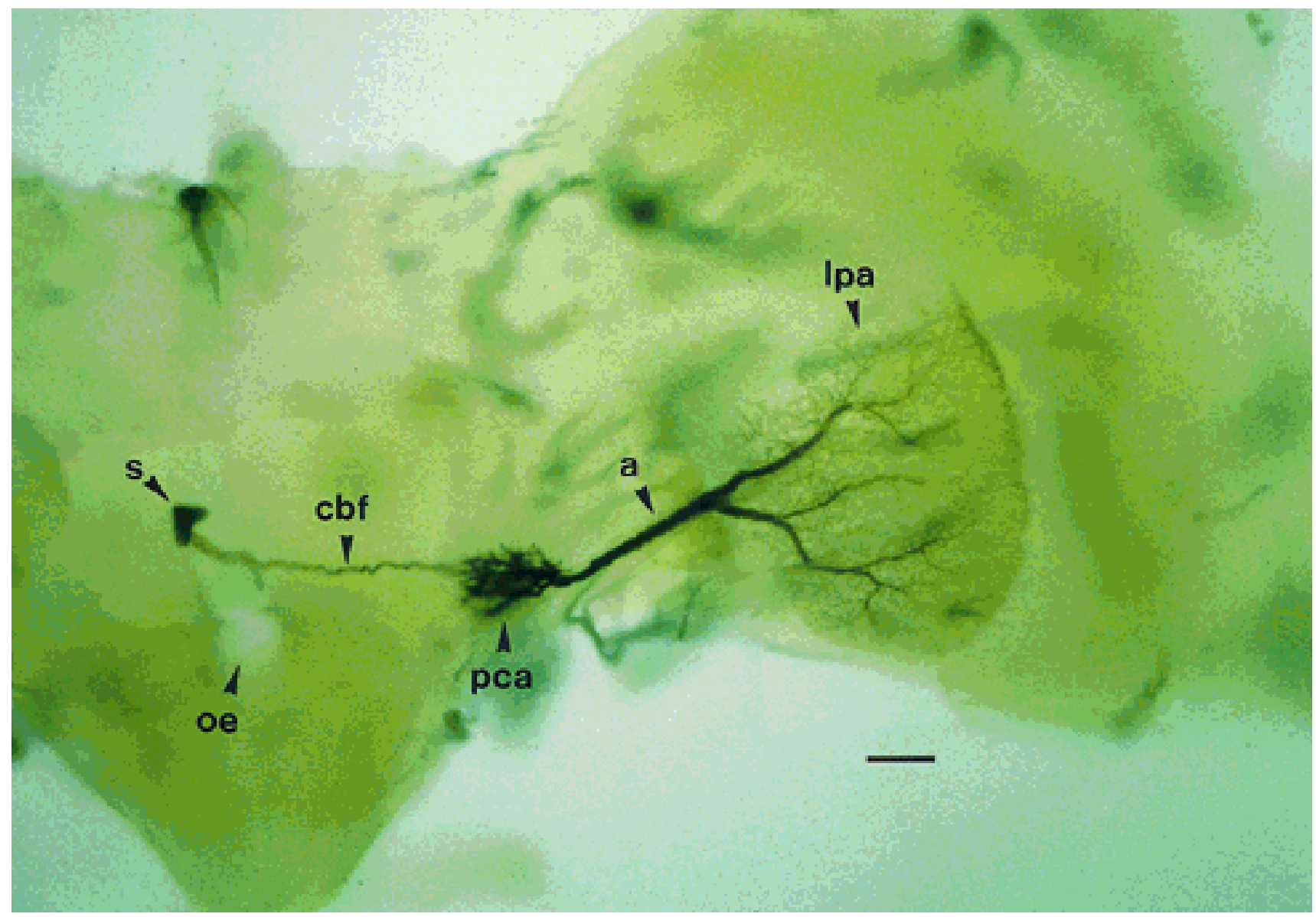

Fig. 3. A montage of light micrographs showing the structure of VCH stained with horseradish peroxidase (HRP). The photographs were made of a whole mount of the fly brain from the back site. The dorsal site of the brain is at the top, and the right site of the brain is at

identical with the element 2 . The last micrograph of this series shows the other end of the presynaptic ribbon, where the contact area between $\mathrm{VCH}$ and the presynaptic site is enlarged. Instead of element 1, a new element (arrowhead 4) is in contact with the presynapse. The contact area size between element 3 and the presynaptic site is enlarged. This example (Fig. 4C-E) represents clear evidence for at least four unstained postsynaptic elements that share common synaptic input with $\mathrm{VCH}$. Because it is technically difficult to trace all postsynaptic elements to a presynaptic site, it cannot be determined whether the number of postsynaptic elements at input synapses of $\mathrm{VCH}$ in the lobula plate is al ways five. There are several candidates for input elements (see Discussion), and it is conceivable that the synaptic organization differs among these input el ements. We never found more than one arbor of $\mathrm{VCH}$ that is postsynaptic to a particular presynaptic site in the lobula plate. Most input elements of $\mathrm{VCH}$ in the lobula plate contain relatively few synaptic vesicles.

Examples of output synapses of $\mathrm{VCH}$ in the lobula plate are presented in Figure 4F,G. In both micrographs a presynaptic ribbon (arrowhead) lies inside a stained crosssection of $\mathrm{VCH}$, indicating an output synapse $(\mathrm{O})$ of $\mathrm{VCH}$. the right of the photograph. HRP was injected in the axon. oe, oesophagus penetration site; s, soma; cbf, cell body fibre; pca, protoce rebrum arborisation; a, axon; Ipa, Iobula plate arborisation. Scale bar $=100 \mu \mathrm{m}$.

Despite the overall weak staining of the arbor of $\mathrm{VCH}$ in Figure 4G, the presynaptic ribbon is dark-labeled by DAB reaction product. We observed the dark appearance of synaptic ribbons in all stained cells but never in unstained cells. It may be due to a strong adherence of HRP to the ribbon proteins. For this reason, we had to take care not to stain cells too strongly to avoid covering the presynaptic ribbons inside $\mathrm{VCH}$. Injection times from 3 to 4 minutes of $1 \mathrm{nA}$ constant current (see Material and Methods) were ideal for our purposes. The number of elements we observed postsynaptically to $\mathrm{VCH}$ in the lobula plate varied between two and four. For the same reason as already mentioned for the input synapses of $\mathrm{VCH}$, we are not able to decide whether there exists a typical fixed synaptic organization at output synapses of $\mathrm{VCH}$. Figure $4 \mathrm{H}, \mathrm{I}$ provides examples for the spatial relationship between input and output synapses in the lobula plate arborisation of $\mathrm{VCH}$. Figure $4 \mathrm{H}$ shows an input synapse (I) and an output synapse $(\mathrm{O})$ of $\mathrm{VCH}$ at a distance of approximately $1 \mu \mathrm{m}$ to each other. A further example of this close spatial relation of input and output synapses is presented in Figure $4 \mathrm{I}$, where both sites are $1.5 \mu \mathrm{m}$ apart. We found this arrangement of input and output synapses to occur not as 

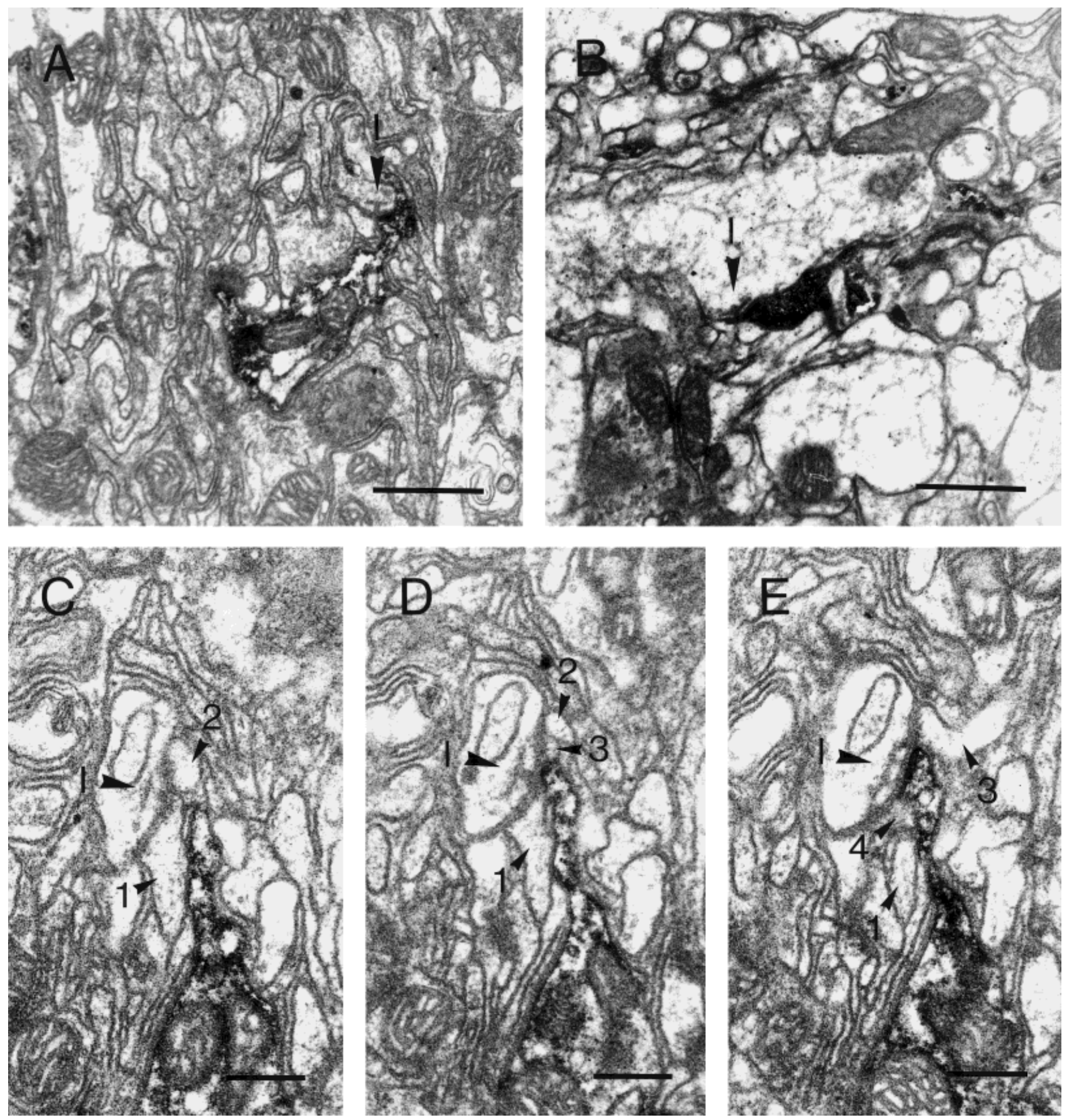

Fig. 4. Electron micrographs of the lobula plate arborisation of three HRP-stained VCHs. A-E: Input synapses (I) of $\mathrm{VCH}$ were identified by the typical T-shaped presynaptic ribbons (arrowhead). Postsynaptically, there are one and two other unstained postsynaptic elements in A and B, respectively. The sections C,D,E are $60-80 \mathrm{~nm}$ apart from each other, and together show probably all other unstained postsynaptic el ements (numbered arrowheads) of one particular input synapse (I) of VCH. F,G: Output synapses (O) of VCH identified by presynaptic ribbons (arrowhead) inside stained arbors of $\mathrm{VCH} . \mathbf{H , I}$ : Spatial relation between input synapses (I) and output synapses $(\mathrm{O})$ of the lobula plate arborisation of $\mathrm{VCH}$. J : Reciprocal connection be tween $\mathrm{VCH}$ and an unstained neuron in the lobula plate. Scale bars = $0.5 \mu \mathrm{m}$ in $\mathrm{A}, \mathrm{B}, \mathrm{F}, \mathrm{G}, \mathrm{H}$, and $\mathrm{I}, 0.5 \mu \mathrm{m}$ in $\mathrm{C}, \mathrm{D}, \mathrm{E}$, and $\mathrm{J}$. frequently as input and output synapses alone, but this may be due to the low probability to hit the appropriate section plane. In one case, we found a reciprocal synaptic connection between $\mathrm{VCH}$ and another neuron in the lobula plate (Fig. 4J ). The presynaptic ribbon inside VCH (arrow- head 0 ) indicating an output synapse $(0)$ is in contact with the same el ement that makes an input synapse (I ) on VCH. Both synaptic sites are only $0.25 \mu$ m apart.

Photomicrographs of the protocerebrum are shown in Figure 5. As in the lobula plate, presynaptic ribbons 

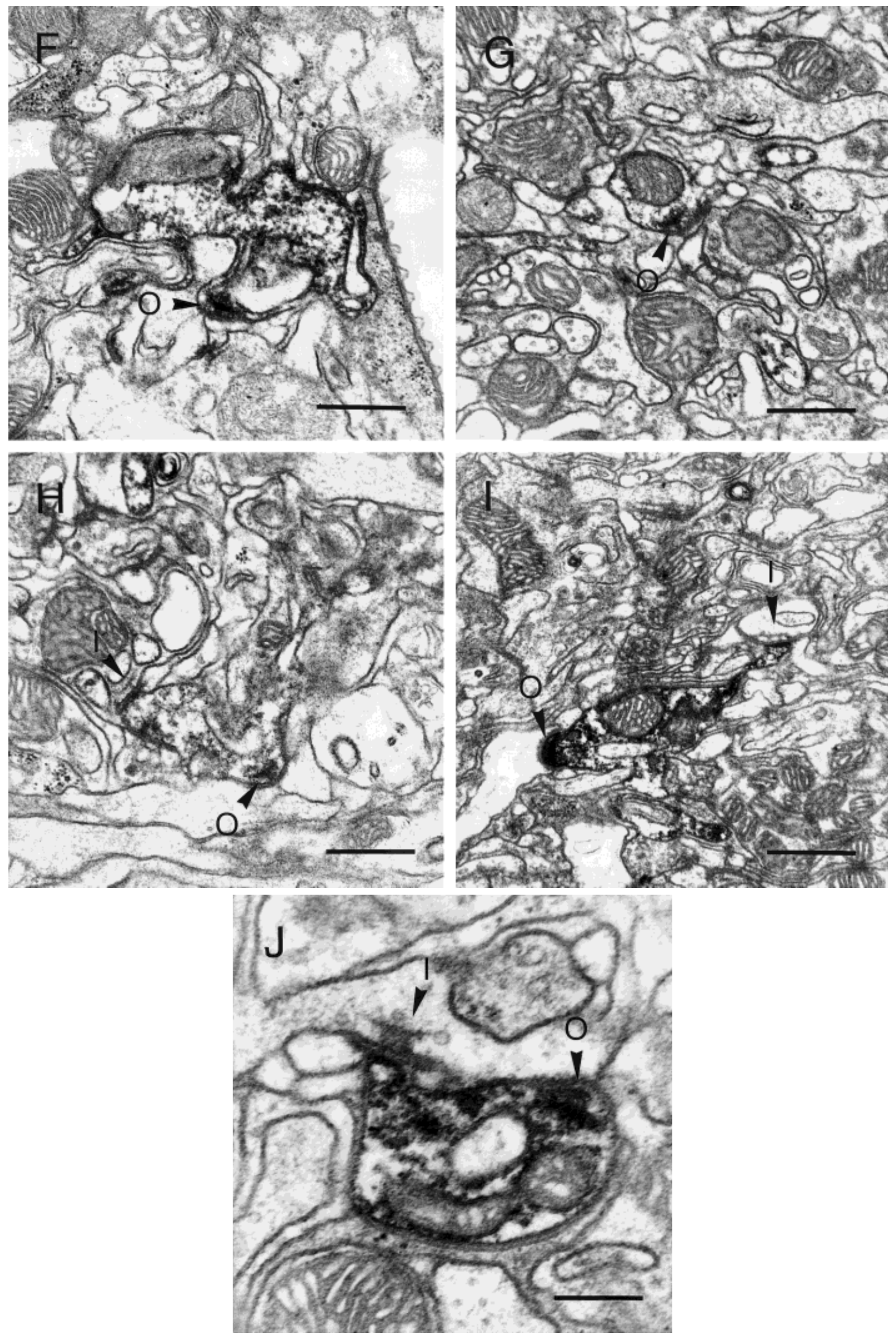

Figure 4 (Continued.) 
(arrowheads) in close contact to stained arbors indicate input synapses (I) of $\mathrm{VCH}$. In Figure $5 \mathrm{~A}$, five cross-sections of $\mathrm{VCH}$ are shown that contact an input element. Two of these five cross-sections receive input at the same synapse. Two input elements of $\mathrm{VCH}$ are visible in Figure 5B. The input element in the left corner of Figure $5 \mathrm{~B}$ has contact with three cross-sections of $\mathrm{VCH}$ and makes synaptic contact with two of them. Furthermore, there are three projections of $\mathrm{VCH}$ inside this input element. Figure 5C,D shows how arbors of $\mathrm{VCH}$ grasp around the input elements, thereby covering the input elements al most totally. In both micrographs only one input synapse is visible, but the tight enclosing of the input elements by arbors of $\mathrm{VCH}$ highly suggests that the other $\mathrm{VCH}$ cross-sections receive input from the same elements. The number of postsynaptic elements we found at input synapses of VCH in the protocerebrum ranged from two to four. We have not found any output synapses of VCH in the protocerebrum. However, we cannot exclude the existence of output synapses, because the protocerebral profiles were mostly darkerlabeled than those of the lobula plate and output synapses might have been covered. In general, the density of $\mathrm{VCH}$ arbors is very high in the protocerebrum compared with the Iobula plate. Furthermore, in the protocerebrum we found multiple synaptic contact sites between one particular input element and profiles of $\mathrm{VCH}$. This arrangement has never been observed in the lobula plate.

\section{DISCUSSION}

We investigated the distribution of synapses in $\mathrm{VCH}$, a motion-sensitive, nonspiking inhibitory interneuron of the third visual neuropil (lobula plate) of the blowfly C. erythrocephala. The inhibitory action of VCH has been suggested to be decisive for the characteristic property of FD1 (Warzecha et al., 1993), an output neuron of the Iobula plate that responds best to small-moving stimuli (Egelhaaf, 1985c).

Our electron microscopic results show that $\mathrm{VCH}$ has input and output synapses close together $(0.5-1.5 \mu \mathrm{m})$ in its main arbor in the lobula plate. By contrast, $\mathrm{VCH}$ appears to have only input synapses in its protocerebral field of neurites. These studies suggest a particular inputoutput organization of $\mathrm{VCH}$ that might be important for the response tuning of FD1 to small-moving objects.

\section{Connectivity between VCH and FD 1}

The presence of output synapses in the Iobula plate arborisation and the possible absence of output synapses in the protocerebral arborisation of $\mathrm{VCH}$ indicate that inhibition of FDI by $\mathrm{VCH}$ probably takes place in the Iobula plate, where the arborisations of both neurons overlap. In principle, inhibition of FD1 could be accomplished directly by $\mathrm{VCH}$ or indirectly by inhibition of the retinotopic input elements feeding onto FD1. Because FD1 is difficult to impale intracellularly, we did not succeed in clarifying whether VCH inhibits FDI directly by using double labeling. If both cells share common retinotopic inputs, inhibition of the retinotopic inputs to FDI by VCH should result in reciprocal synaptic complexes between $\mathrm{VCH}$ and these input elements. We found one example of a reciprocal synaptic arrangement between $\mathrm{VCH}$ and an unidentified el ement (Fig. 4J ).

\section{Input elements to VCH}

Like other tangential neurons, $\mathrm{VCH}$ receives excitatory and inhibitory synaptic input (Borst and Egelhaaf, 1990; Borst et al., 1995) from retinotopically arranged (E gel haaf et al., 1993) ipsilateral motion-sensitive elements. Columnar elements originating in the medulla (the T4 and $Y$ cells) and in the lobula (T5 cells) terminate in the lobula plate (Strausfeld, 1984; Fischbach and Dittrich, 1989; Strausfeld and Lee, 1991; Bausenwein et al., 1992). These neurons are likely to represent the retinotopic input of the tangential neurons in the lobula plate. T4 cells have been shown by electron microscopy to be presynaptic to an HS cell (Strausfeld and Lee, 1991). Most elements we found presynaptically to $\mathrm{VCH}$ in the lobula plate had only few synaptic vesides (Fig. 4). Presynaptic elements containing many vesicles were rare (data not shown).

VCH responds to visual motion in its preferred direction or in its null direction in front of the contralateral eye with EPSPs or IPSPS, respectively (Fig. 1). EPSPs with large and small amplitudes are visible in Figure 1. The amplitude spectrum (Fig. 2A) shows two amplitude groups, suggesting the existence of two excitatory contralateral input neurons. This is in accordance with findings of Hausen (1977) for unidentified $\mathrm{CH}$ neurons (VCH or DCH). Hausen (1977) recorded simultaneously from an unidentified $\mathrm{CH}$ and the contralateral $\mathrm{H} 2$ as well as from $\mathrm{DCH}$ and the contralateral $\mathrm{Hl}$. Large EPSPs of the unidentified $\mathrm{CH}$ were correlated with action potentials of $\mathrm{H} 2$, and small EPSPs of DCH were correlated with action potentials of $\mathrm{H} 1$ (Hausen, 1977). Whether this is the same for $\mathrm{VCH}$ has to be shown in further experiments. The contralateral $\mathrm{H} 1$ terminates in the lobula plate, and the contralateral $\mathrm{H} 2$ terminates in the protocerebrum. The frequency spectrum of IPSPs (Fig. 2B) suggests the existence of one inhibitory contralateral input element. This inhibitory element is hitherto unknown. Visual motion in front of the ipsilateral eye elicits graded shifts of the membrane potential and stimulus-correlated fluctuations of the membrane potential (Fig. 1). EPSPs cannot be discriminated as clearly as during contralateral stimulation (Fig. 1). This phenomenon was first noted by Hausen (1976, 1977). The difference between the responses of VCH to contralateral and ipsilateral visual stimulation is likely to have two reasons. First, the contralateral input is conveyed by only three spiking neurons (two excitatory and one inhibitory), whereas the ipsilateral input is mediated by several hundred neurons that probably do not spike in synchrony or do not spike at all. Second, by comparing the micrographs of the lobula plate and the protocerebrum in Figure 4, one could speculate that synaptic input from neurons reaching VCH in the protocerebrum (maybe the contralateral H2) could be amplified by multiple input synapses between both neurons. In the lobula plate, wealways found only one $\mathrm{VCH}$ arbor postsynaptic to a particular input synapse. In contrast, in the protocerebrum, VCH arbors receive multiple synapses from one presynaptic el ement and often two VCH arbors are postsynaptically at the same presynaptic site.

\section{E lements sharing synaptic input with VCH}

We found up to four unknown profiles at any given synaptic site in the lobula plate that shares the same input element as VCH (Fig. 4C-E). The dendritic trees of the following tangential cells could receive input at the same 

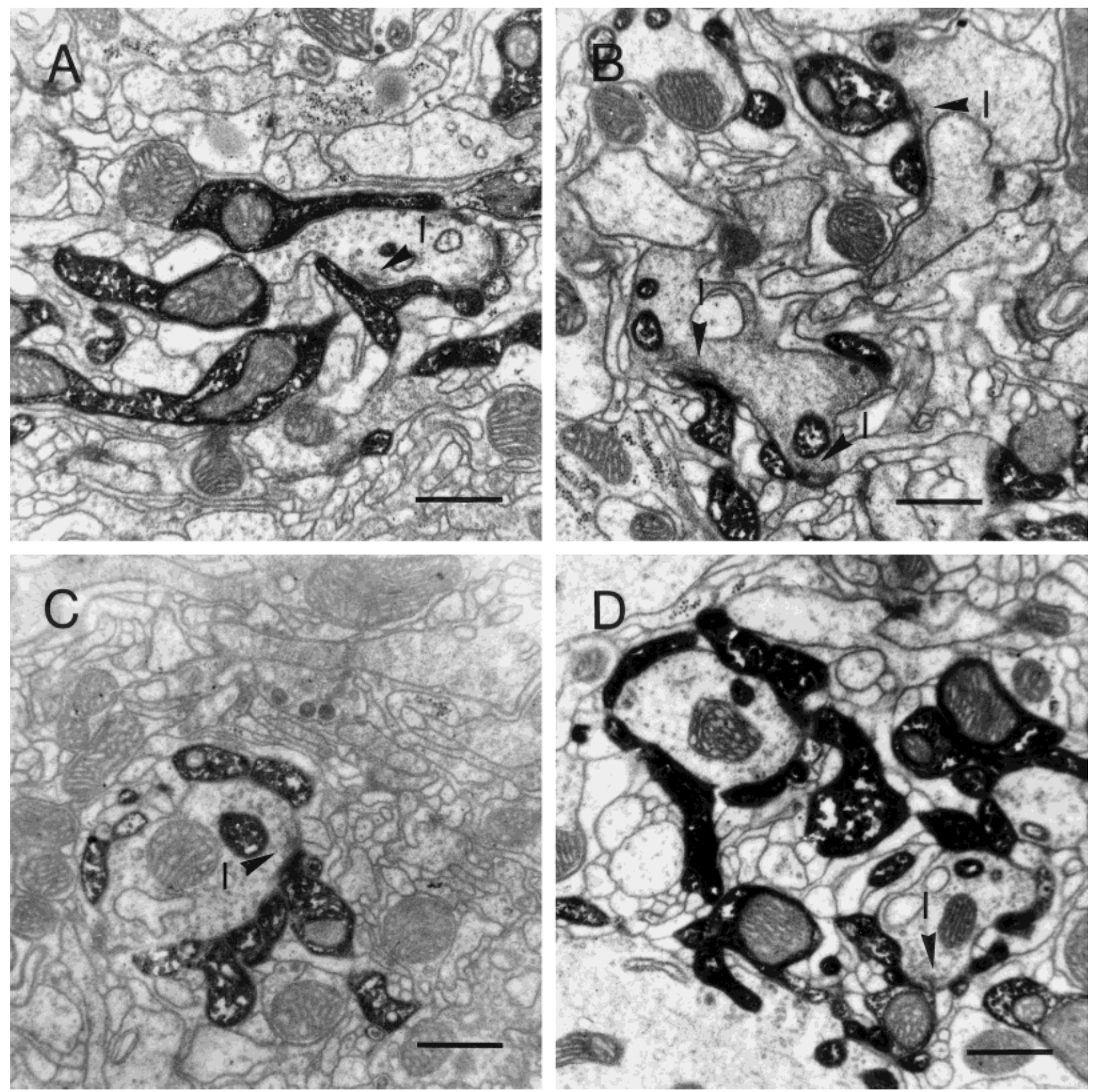

Fig. 5. Electron micrographs of the protocerebral arborisation of one HRP-stained VCH. We found input synapses (I) but no output synapses of $\mathrm{VCH}$ in the protocerebrum. One input synapse can contact two (A) or more (not shown) arbors of $\mathrm{VCH}$. One input neuron can

synapses as VCH: FD1 in the distal part of the lobula plate, FD4 (Egelhaaf, 1985c) south horizontal (HSS) in the ventral lobula plate, equatorial horizontal (HSE) in the medial Iobula plate, $\mathrm{H} 1$, and $\mathrm{H} 2$ (Hausen, 1984). The unknown postsynaptic elements could also belong to local interneurons of the lobula plate, about which little is known. Furthermore, there are reports about reciprocal synaptic contacts between neighboring terminals of T4 neurons in the lobula plate (Strausfeld and Lee, 1991). At input synapses of VCH in the dorsal protocerebrum, we found up to three un-

make several input synapses on arbors of VCH (B). Input neurons are often tightly surrounded by the stained profiles of VCH $(\mathbf{C}, \mathbf{D})$. Scale bar $=0.5 \mu \mathrm{m}$

known elements that share the same input as $\mathrm{VCH}$. These elements could belong to HSE and to north horizontal (HSN), which are known to receive (like $\mathrm{VCH}$ ) excitatory input from the contralateral elements (Hausen, 1982). Double recordings revealed that HSN receives input from the contralateral $\mathrm{H} 2$ in the protocerebrum (Haag, 1994).

\section{E lements postsynaptic to VC H}

The number of elements found to be postsynaptic to $\mathrm{VCH}$ in the lobula plate at a given synaptic site ranges 
from two to four. Could the inhibition of all these postsynaptic elements be involved in the small field tuning of FD1? We do not know whether all postsynaptic elements are different neurons. If FDI is directly inhibited by VCH, the synaptic gain could be raised if several FDl arbors receive input from the same presynaptic site. If the postsynaptic elements represent different neurons, one may ask what function the inhibitory action of $\mathrm{VCH}$ might have beside the small field tuning of FD1. If FD1 is indirectly inhibited by $\mathrm{VCH}$ via its retinotopic input elements, the postsynapticelements could betheretinotopically arranged input elements T5 and/or T4 and/or Y. Because it is likely that several tangential neurons receive input from the same retinotopic elements, it is possible that their inhibition by $\mathrm{VCH}$ could also affect the retinotopic input of other tangential cells. This has to be investigated in further experiments. If this is not the case, we have to assume that the terminal arborisations of the retinotopic elements are functionally separated. A note in Hausen (1981) suggests that VCH also inhibits $\mathrm{H} 1$.

\section{F unctional implications of colocalization of input and output synapses}

The close proximity of input and output synapses we found in the arborisation of $\mathrm{VCH}$ in the lobula plate raises the question of the functional significance of this particular wiring scheme. This point might be of general relevance, because nonspiking local interneurons like $\mathrm{VCH}$ with input and output synapses in their dendritic trees have been found in several other systems, such as amacrine cells of the vertebrate retina (Dowling and Boycott, 1966), mitral and granule layer cells of the vertebrate olfactory bulb (Rall et al., 1966; Rall and Shepherd, 1968), relay neurons of the cat lateral geniculate nucleus (Hamos et al., 1985), and nonspiking interneurons of the crayfish (Kondoh and Hisada, 1986) and the locust (Wilson and Phillips, 1982; Watson and Burrows, 1988). If we take into account that $\mathrm{VCH}$ receives retinotopic input from the ipsilateral eye, an obvious effect of the close location of input and output synapses in $\mathrm{VCH}$ is that the retinotopic activity pattern is spatially more or less conserved in the inhibitory output pattern of $\mathrm{VCH}$. How well this topography is conserved depends on the spread of the electric activity in the lobula plate arborisation of $\mathrm{VCH}$ and on the properties of synaptic transmitter release at these synapses. If the output synapses are electronically far away from each other but each output synapse is close to an input synapse, $\mathrm{VCH}$ would convert an excitation in an inhibition at many locally restricted sites in the lobula plate. The passive membrane properties of $\mathrm{VCH}$, revealed by electrophysiologic investigations and compartmental modeling (Borst and Haag, 1996), suggest that the spread of excitation within the $\mathrm{VCH}$ arbor will result in a spatially blurred output. This is to say that $\mathrm{VCH}$ acts as a low-pass spatial filter. Thus, visual stimulus patterns with low spatial frequencies would be translated accurately in an inhibiting output pattern, whereas high-frequency visual stimuli would lead to a spatially uniform inhibition pattern. Imaging studies of either presynaptic calcium or membrane potential in the lobula plate arborisation of $\mathrm{VCH}$ with high temporal and spatial resolutions might help determine whether the inhibitory output pattern of $\mathrm{VCH}$ is spatially blurred with respect to the retinotopic input pattern.
The function of calcium in the lobula plate arborisation of $\mathrm{VCH}$ might also not be restricted to transmitter release. This idea is supported by the finding that other tangential cells that have no output synapses in the lobula plate (Hausen et al., 1980) nevertheless possess voltagedependent calcium channels in their dendrites (Egel haaf and Borst, 1995). Besides calcium channels, VCH also possesses voltage-dependent potassium channels (Haag and Borst, 1996). The functional implications of these active membrane properties, besides for transmitter release, are not yet understood. In this respect, the synaptic organization of $\mathrm{VCH}$ might providea hint. The closeness of input and output synapses in the lobula plate arbor of VCH results in a very fast signal conduction without the need for active membrane properties. Ther efore, one could speculate that the active membrane properties in VCH will primarily affect the spatial spread of excitation instead of the temporal processing of input signals (Haag and Borst, 1996).

Another point of view concerning the active membrane properties comes from model simulations in which an expansive nonlinearity was found to be decisive for the generation of small field tuning (Reichardt et al., 1983; Egel haaf, 1985a; Borst and Egelhaaf, 1993). These nonlinearities were realized by an appropriate synaptic transfer function either between VCH and FDI (Borst and Egelhaaf, 1993) or between the motion-sensitive input elements and FD1 (Reichardt et al., 1983; Egel haaf, 1985a). The cellular mechanisms underlying such a hypothetical nonlinearity were not specified. It has been suggested that the active membrane properties of $\mathrm{VCH}$ might play a role in the generation of such a nonlinear transfer function (Borst and Egel haaf, 1993).

An additional point of view comes from a study of the inhibitory interneurons in the thoracic ganglion of the locust (Laurent, 1993) that resemble VCH, in that input and output synapses are intermingled in their dendritic trees (Watson and Burrows, 1988) and in that they possess voltage-gated potassium and calcium channels (Laurent, 1993). These neurons transfer presynaptic spikes in postsynaptic IPSPs of al most constant amplitude irrespective of their own membrane potential. This property is due to the activity of their voltage-gated channels (Laurent, 1993). Assuming the same for $\mathrm{VCH}$, this would realize a linear transfer function between its synaptic input and output in the whole lobula plate arbor irrespective of potential differences due to its retinotopic input-output organization and large receptivefield. Further electrophysiologic investigations are necessary to understand the functional relationship between anatomy and physiology of $\mathrm{VCH}$.

\section{ACKNOWLE DGME NTS}

This work was started at the MPI of Biological Cybernetics. We thank K.G. Götz for support in the early stage of this work, K. Kirschfeld for permission to use the electron microscope in his laboratory, and A. Münster for helpful tips concerning electron microscopic procedure.

\section{LITERATURE CITED}

Bausenwein, B., A.P.M. Dittrich, and K.F. Fischbach (1992) The optic lobe of Drosophila melanogaster: II. Sorting of retinotopic pathways in the medulla. Cell Tissue Res. 267:17-28. 
Borst, A., and M. Egelhaaf (1990) Direction selectivity of the blowfly motion-sensitive neurons is computed in a two-stage process. Proc Natl. Acad. Sci. USA 87:9363-9367.

Borst, A., and M. Egelhaaf (1992) In vivo imaging of calcium accumulation in fly interneurons as elicited by visual motion stimulation. Proc. Natl. Acad. Sci. USA 89:4139-4143.

Borst, A., and M. Egelhaaf (1993) Processing of synaptic signals in fly visual interneurons selectively responsive to small moving objects. In A Aertsen (ed): Brain Theory. Amsterdam: Elsevier Science Publishers B.V., pp. 47-66.

Borst, A., and J . Haag (1996) The intrinsic electrophysiological characteristics of fly lobula plate tangential cells: I. Passive membrane properties. J. Computat. Neurosci. 3:313-336.

Borst, A., M. Egelhaaf, and J. Haag (1995) Mechanisms of dendritic integration underlying gain control in fly motion-sensitive interneurons. J . Computat. Neurosci. 2:5-18.

Burkhardt, W., and V. Braitenberg (1976) Some peculiar synaptic complexes in the first visual ganglion of the fly, Musca domestica. Cell Tissue Res. 173:287-308.

Campos-Ortega, J .A., and N.J . Strausfeld (1973) Synaptic connections of intrinsic cells and basket arborizations in the external plexiform layer of the flye's eye. Brain Res. 59:119-136.

Dowling, J .E., and B.B. Boycott (1966) Organization of the primate retina: Electron microscopy. Proc. R. Soc. Lond. B. 166:80-111.

Eckert, H., and K. Meller (1981) Synaptic structures of identified, motionsensitive interneurones in the brain of the fly, Phaenicia. Verh. Dtsch. Zool. Ges. Stutlgart: Gustav Fischer Verlag, pp. 179. Abstr.

Eckert, H., and D.R. Dvorak (1983) The centrifugal horizontal cells in the Iobula plate of the blowfly, Phaenicia sericata. J. Insect Physiol. 29:547-560.

Egelhaaf, M. (1985a) On the neuronal basis of figure-ground discrimination by relative motion in the visual system of the fly: III. Possible input circuits and behavioural significance of the FD-cells. Biol. Cybern. 52:267-280.

Egelhaaf, M. (1985b) On the basis of figure-ground discrimination by relative motion in the visual system of the fly: I. Behavioral constraints imposed on the neuronal network and the role of the optomotor system. Biol. Cybern. 52:123-140.

Egelhaaf, M. (1985c) On the basis of figure-ground discrimination by relative motion in the visual system of the fly: II. Figure-detection cells, a new class of visual interneurones. Biol. Cybern. 52:195-209.

Egelhaaf, M. (1987) Dynamic properties of two control systems underlying visually guided turning in house-flies. J . Comp. Physiol. A. 161:777-783.

Egelhaaf, M. (1990) Spatial interactions in the fly visual system leading to selectivity for small-field motion. Naturwissenschaften 77:182-185.

Egelhaaf, M., and A. Borst (1995) Calcium accumulation in visual interneurones of the fly: Stimulus dependence and relationship to membrane potential. J . Neurophysiol. 73:2540-2552.

Egelhaaf, M., K. Hausen, W. Reichardt, and C. Wehrhahn (1988) Visual course control in flies relies on neuronal computation of object and background motion. TINS 11:351-358.

Egelhaaf, M., A. Borst, A.K. Warzecha, S. Flecks, and A. Wildemann (1993) Neural circuit tuning fly visual neurons to motion of small objects: II. I nput organization of inhibitory circuit elements revealed by electrophysiological and optical recording techniques. J . Neurophysiol. 69:340-351.

Fischbach, K.F., and A.P.M. Dittrich (1989) The optic lobe of Drosophila melanogaster: I. A Golgi analysis of wild-type structure. Cell Tissue Res. 258:441-475.

Franceschini, N., and K. Kirschfeld (1971) Les phenomenes de pseudopupille dans I'oeil compose de drosophila. Kybernetik 9:159-182.

Haag, J . (1994) Aktive und passive membraneigenschaften bewegung sempfindlicher interneurone der schmeissfliege Calliphora erythrocephala. 1-115. Doctoral thesis, Tübingen: Eberhard-Karls-Univeristāt.

Haag, J ., and A. Borst (1996) Frequency-dependent amplification of synaptic input by active dendritic membrane processes in motion-sensitive interneurons. Nature 379:639-641.
Hamos, J.E., S.C. Van Horn, D. Raczkowski, D.J. Uhlrich, and S.M. Sherman (1985) Synaptic connectivity of a local circuit neurone in lateral geniculate nucleus of the cat. Nature 317:618-621.

Hausen, K. (1976) Functional characterisation and anatomical identification of motion sensitive neurons in the lobula plate of the blowfly Calliphora erythrocephala. Z. Naturforsch. 31:629-633.

Hausen, K. (1977) Struktur, funktion und konnektivitaet bewegung sempfindlicher interneuroneim dritten optischen neuropil der schmeissfliege Calliphora erythrocephala. 1-254. Doctoral thesis, Tübingen: Eberhard-Karls-Universitāt.

Hausen, K. (1981) Monocular and binocular computation of motion in the Iobula plate of the fly. In Verh. Dtsch. Zool. Ges. 1981. Stuttgart: Gustav Fischer Verlag, pp. 49-70.

Hausen, K. (1982) Motion sensitive interneurons in the optomotor system of the fly: I. The horizontal cells: Structure and signals. Biol. Cybern. 45:143-156.

Hausen, K. (1984) The Iobula-complex of the fly: Structure, function and significance in visual behaviour. In M.A. Ali (ed): Photoreception and Vision in Invertebrates. New York: Plenum, pp. 523-559.

Hausen, K., K. Wolburg-Buchholz, and W.A. Ribi (1980) The synaptic organisation of visual interneurons in the lobula complex of flies. Cell Tissue Res. 208:371-387.

Kondoh, Y., and M. Hisada (1986) Distribution and ultrastructure of synapses on a premotor local nonspiking interneurone of the crayfish. J . Comp. Neurol . 254:259-270.

Laurent, G. (1993) A dendritic gain control mechanism in axonless neurons of the locust, Schistocerca americana. J . Physiol. 470:45-54.

Meyer, E.P., C. Matute, P. Streit, and D.R. Nässel (1986) Insect optic lobe neurons identifiable with monocl onal antibodies to GABA. Histochemistry 84:207-216.

Miller, J .P., and A.I . Selverston (1979) Rapid killing of single neurons by irradiation of intracellularly injected dye. Science 206:702-704.

Rall, W., and T.S. Shepherd (1968) Theoretical reconstruction of field potentials and dendrodendritic synaptic interactions in olfactory bulb. J . Neurophysiol. 31:884-915.

Rall, W., T.S. Shepherd, T.S. Reese, and M.W. Brightman (1966) Dendrodendritic synaptic pathway for inhibition in the olfactory bulb. Exp. Neurol. 14:44-56.

Reichardt, W., T. Poggio, and K. Hausen (1983) Figure-ground discrimination by relative movement in the visual system of the fly: Part II. Towards the neural circuit 1. Biol. Cybern. 46:1-30.

Reichardt, W., M. Egelhaaf, and A. Guo (1989) Processing of figure and background motion in the visual system of the fly. Biol. Cybern 61:327-345.

Shaw, S.R., and I.A. Meinertzhagen (1986) Evolutionary progression at synaptic connections made by identified homologous neurones. Proc Natl. Acad. Sci. USA 83:7961-7965.

Strausfeld, N.J . (1984) Functional neuroanatomy of the blowfly's visual system. In M.A. Ali (ed): Photoreception and Vision in Invertebrates. New York: Plenum, pp. 483-522.

Strausfeld, N.J ., and J.K. Lee (1991) Neuronal basis for parallel visual processing in the fly. Vis. Neurosci. 7:13-33.

Warzecha, A.K., M. Egelhaaf, and A. Borst (1993) Neural circuit tuning fly visual interneurons to motion of small objects: I. Dissection of the circuit by pharmacological and photoinactivation techniques. J . Neurophysiol. 69:329-339.

Watson, A.H.D., and M. Burrows (1988) Distribution and morphology of synapses on nonspiking local interneurones in the thoracic nervous system of the locust. J . Comp. Neurol . 272:605-616.

Wilson, J .A., and C.E. Phillips (1982) Locust non-spiking interneurons which tonically drive antagonistic motor neurons: Physiology, morphology and ultrastructure. J . Comp. Neurol. 204:21-31. 